\title{
Applied Fluid-Structure Interaction Technique to Initial Insight into the Effect of Exercise on the Aortic Valve Stroke Work
}

\author{
Bahraseman $\mathrm{HG}^{1 *}$, Languri EM², Espino $\mathrm{DM}^{3}$, Shojaei $\mathrm{H}^{4}$, Hassani $\mathrm{K}^{5}$ and Derakhshandeh $\mathrm{H}^{6}$ \\ ${ }^{1}$ Department of Mechanical Engineering, California State Polytechnic University, Pomona, CA, USA \\ ${ }^{2}$ Department of Mechanical Engineering, Tennessee Technological University, Cookeville, TN, USA \\ ${ }^{3}$ School of Mechanical Engineering, University of Birmingham, Birmingham, England, UK \\ ${ }^{4}$ Department of Pathology, University Hospitals Case Medical Center, Case Western Reserve University, Cleveland, OH, USA \\ ${ }^{5}$ Department of Biomechanics, Science and Research Branch, Islamic Azad University, Tehran, Iran \\ ${ }^{6}$ Department of Mechanical and Material Engineering, University of Nebraska-Lincoln, Lincoln, NE, USA
}

\begin{abstract}
The left ventricular stroke work is a measure of the work done by the left ventricle during the ejection of blood throughout per cardiac cycle. The aim of this investigation was to propose a model to numerically evaluate the stroke work for a healthy subject by using a fluid-structure interaction (FSI) simulation during exercise protocol. Aortic valve dimensions were calculated using an imaging technique of echocardiography. An FSI simulation was performed using an Arbitrary Lagrangian-Eulerian (ALE) mesh. Boundary conditions were defined by pressure loads on ventricular and aortic sides. Stroke work was predicted to increase to $121 \%$ from $60 \mathrm{bpm}$ to $125 \mathrm{bpm}$, and it did not increase much above $125 \mathrm{bpm}$. Based on derived regression equations of our FSI results for stroke work and comparing of them with clinical ones, numerically-predicted stroke work values are in good agreements with published clinical data. The slope of stroke work changes to mean arterial pressure, while exercise protocol, is $168.08 \mathrm{ml}$ which is $12.2 \%$ less than the average slope of clinical data. The $y$-axis intercept of stroke work changes to mean arterial pressure, while exercise protocol, is $-11186 \mathrm{mmHg} . \mathrm{ml}$ which is $15 \%$ less than the average $y$-axis intercept of clinical data. Our results for the specific patient show that numerical methods can be proposed to predict good estimates of patient specific stroke work at different heart rates.
\end{abstract}

Keywords: Aortic valve; Finite element method; Fluid solid interaction; Stroke work

\section{Introduction}

Measurement of stroke work is a crucial factor in understanding the development of heart problems and subsequent clinical diagnosis [1]. For example, the stroke work index is a reliable criterion of left ventricle performance which requires an invasive procedure, and has been usually measured and utilized in the settings of intensive care units and operation rooms [2-4]. While invasive methods have been used to measure stroke work, such techniques are expensive and include risks to patients [5]. Computational procedures, however, have the potential to determine stroke work but bypassing such limitations.

Invasive, and cost intensive techniques have been used so far [5-7]. For example, Loeb et al. assessed left ventricular function after acute myocardial infarction using stroke volume calculation [8]. They plotted left ventricular stroke-work index (LVSWI) versus left ventricular end-diastolic pressure (LVEDP). Linhart investigated pacing-induced alterations in stroke volume in the appraisal of myocardial function [9]. They performed right atrial pacing in 16 subjects while right and left heart catheterizations. They concluded when pacing ventricular function curves were performed relating left ventricular stroke work (SW) to LVEDP, normal patients demonstrated a steep curve. Hamosh and Cohn studied acute myocardial infarction with measuring left ventricular stroke volume. Hamosh and Cohn [10] in 40 healthy patients estimated accuracy of stroke volume via reservoir pressure concept and three element [11]. Also, due to the fact that invasive experiments could be risky for human subject, there have been some studies on animals; although, no data related to stroke work was found in some of them, but it can be derived from their hemodynamic results [12-18].

Simultaneous simulations of Fluid-Structure Interaction (FSI) have the potential for non-invasive prediction of stroke work. Recently, FSI has been utilized to investigate heart and heart valve mechanics [19-31].
Studies include use of a two-dimensional model to evaluate the stroke volume and cardiac output for a healthy subject [28]. That was done by coupling the FSI simulation with an echo-Doppler method at rest and while exercise. Detailed attention was taken into account to validate the simulation against cardiac function measures that can be reliably computed by using clinical protocols, with varying heart rates [28] Moreover, it was possible to predict the effect of heart rate increment while exercising on blood hemodynamics through the aortic valve, and strains and stresses experienced by the valve leaflets [28]. Developing such models to enable stroke work would potentially be of clinical value because they are supposed to perform it more readily. However, so far FSI and clinical measurements have not been jointed to predict a patient's stroke work. Effect of heart rate changes (e.g. due to exercise) on stroke work of the patient have not been analyzed either. Heart rate would be a significant parameter to consider because it brings about large differences in stroke work.

The aim of this research was to assess the influence of exercise on stroke work at different heart rates from rest to exercise. Using FSI model and our two-dimensional aortic valve geometry, we evaluate stroke work during exercise. The key valves of dimensions and boundary conditions

*Corresponding author: Bahraseman HG, Department of Mechanical Engineering, California State Polytechnic University, Pomona, 91768, CA, USA, Tel: +1 909-869-7659; E-mail: hamidrezag@cpp.edu

Received October 26, 2017; Accepted December 10, 2017; Published December 16, 2017

Citation: Bahraseman HG, Languri EM, Espino DM, Shojaei H, Hassani K, et al. (2017) Applied Fluid-Structure Interaction Technique to Initial Insight into the Effect of Exercise on the Aortic Valve Stroke Work. J Appl Mech Eng 6: 295. doi: $10.4172 / 2168-9873.1000295$

Copyright: (c) 2017 Bahraseman HG, et al. This is an open-access article distributed under the terms of the Creative Commons Attribution License, which permits unrestricted use, distribution, and reproduction in any medium, provided the original author and source are credited. 
Citation: Bahraseman HG, Languri EM, Espino DM, Shojaei H, Hassani K, et al. (2017) Applied Fluid-Structure Interaction Technique to Initial Insight into the Effect of Exercise on the Aortic Valve Stroke Work. J Appl Mech Eng 6: 295. doi: 10.4172/2168-9873.1000295

for our study were acquired from a single volunteer,-enabling an initial assessment of the applicability of the model for personalized healthcare.

\section{Materials and Methods}

\section{Overview}

A healthy human male adult, 33 years old $(80 \mathrm{~kg}, 175 \mathrm{~cm}, \mathrm{BMI}$ : 26.1), participated in this study. His healthy cardiovascular function was confirmed by clinical protocols. The informed consent was obtained. The workflow of the study is provided in Figure 1.

\section{Numerical approach}

Systolic and diastolic pressures were recorded during the test via a brachial pressure cuft (Figure 2). Using echo-cardiography, the aortic valve geometry (Figure 3 ) and its dimensions were obtained (Table 1). The computer aided design (CAD) model of the aortic valve geometry were created with Solidworks package based on the clinicallymeasured data. The leaflet mechanical properties were considered as homogenous, isotropic with linear stress-strain relationship [20-24,32]. Blood was presumed to be Newtonian and an incompressible fluid [33]. All material properties are given in Table $2[34,35]$.

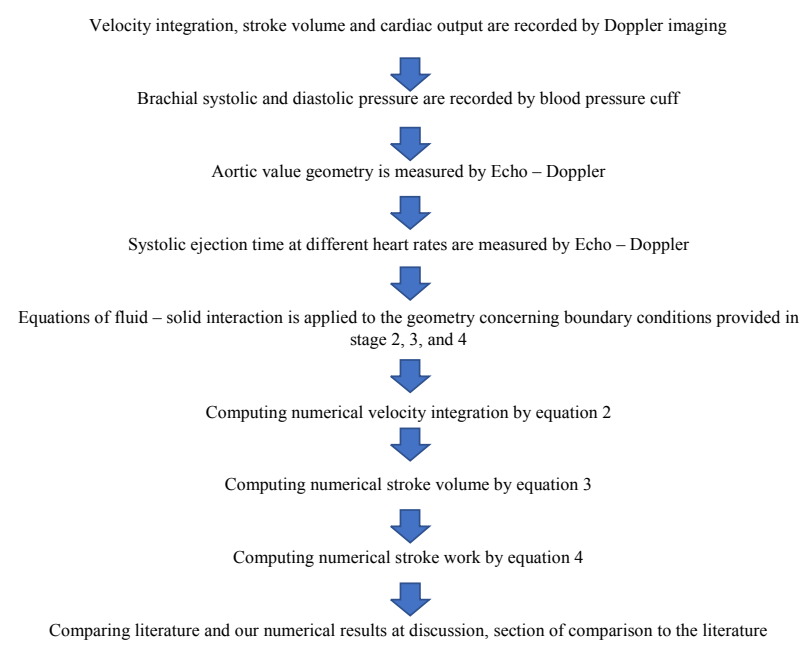

Figure 1: The workflow of the study.

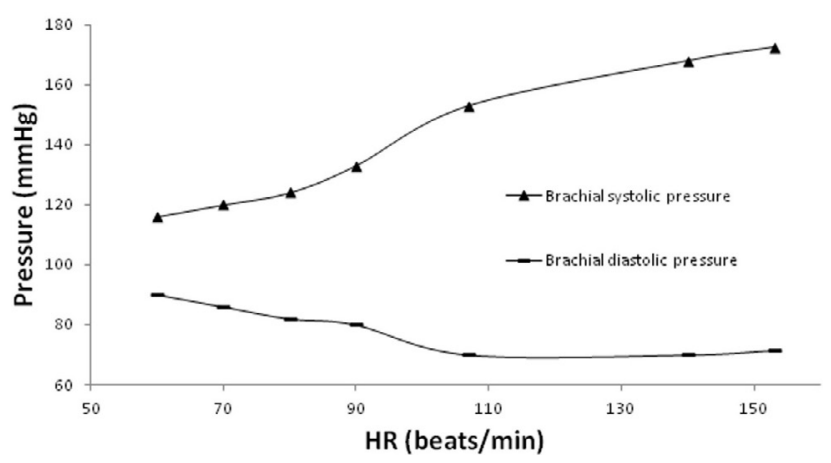

Figure 2: Systolic and diastolic pressures.

\begin{tabular}{|c|c|c|c|}
\hline Viscosity (Pa.s) & Density $\left(\mathbf{k g} / \mathbf{m}^{\mathbf{3}}\right)$ & Young's modulus $\mathbf{( N / \mathbf { m } ^ { 2 } )}$ & Poisson ratio \\
\hline $3.5 \times 10^{-3}$ & 1056 & $6.8 \times 10^{6}$ & 0.49 \\
\hline
\end{tabular}

Table 2: Mechanical properties of fluid and solid.

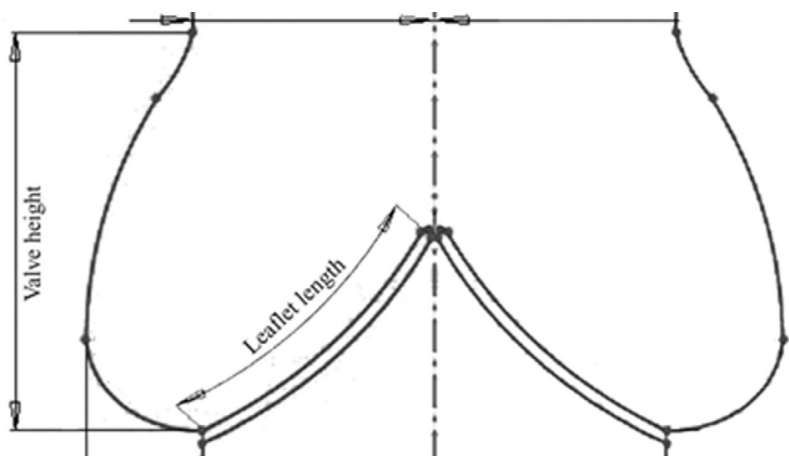

Figure 3: Two-dimentional aortic valve geometry.

The CAD model then discritized and imported into the finite element analysis package, Comsol Multi-physics (v4.2, Comsol Ltd, Cambridgeshire, UK). The fluid and strucure governing equations were applied and the material geometries were introduced. Pressure boundary condition was used at the inflow (the ventricular side) and outflow (the aortic side) boundaries for the fluid mechanics module (Figure 3). The use of a moving ALE mesh enabled the deformation of the fluid mesh to be tracked without the need for re-meshing [32]. The second order Lagrangian elements were utilized to define the mesh. The model is capable to solve time-dependent FSI model $[25,36]$. The model then validated to ensure its independency from mesh resolutions [28].

\section{Analysis of fluid dynamics}

The aortic area was calculated utilizing:

$$
\text { Area }=\pi\left(\frac{D}{2}\right)^{2}
$$

where $\mathrm{D}$ is the calculated ascending aortic diameter after the sinotubular junction (Table 1). For FSI simulations, the mean velocity numerically was obtained at each time step of the ejection period:

$$
\text { Velocity integration }=\phi_{0}^{\text {ejection time }} V . d t
$$

where $V$ is the mean fluid velocity through the outlet boundary. Comparison of measurements of velocity integration, cardiac output and stroke volume enabled quantitative validation of the FSI model.

Knowing the velocity integration and aortic area, one can calculate the strock volume as Equation (5):

Stroke volume $=$ Velocity integration $\times$ Aortic area (3)

The total left ventricular stroke work (SW) is calculated by the product of left ventricular pressur during ejection and the ejected volume of blood integrated over the ejection interval (Figure 3 ).

$$
\text { Strokework }=\int_{0}^{\text {ejectiontime }} \text { Pressure } \times d(\text { strokevolume })
$$

\begin{tabular}{|c|c|c|c|c|c|c|}
\hline $\begin{array}{l}\text { Aortic side radius } \\
\qquad(\mathrm{mm})\end{array}$ & $\begin{array}{l}\text { Leaflet's length } \\
(\mathrm{mm})\end{array}$ & $\begin{array}{l}\text { Ascending aorta radius after } \\
\text { sinotubular junction ( } \mathrm{mm})\end{array}$ & $\begin{array}{l}\text { Valve's height } \\
\text { (mm) }\end{array}$ & $\begin{array}{c}\text { Maximum radius of } \\
\text { normal aortic root }(\mathrm{mm})\end{array}$ & $\begin{array}{c}\text { Leaflet's thickness } \\
(\mathrm{mm})\end{array}$ & $\begin{array}{l}\text { Ventricular side } \\
\text { radius }(\mathrm{mm})\end{array}$ \\
\hline 11.5 & 16.6 & 11.75 & 20.36 & 16.65 & 0.6 & 11.1 \\
\hline
\end{tabular}




\section{Results}

\section{Systolic pressure changes to stroke volume}

Figure 4 provides systolic pressure changes to stroke volume at different heart rates. Overall, in terms of ascending and descending heart rates, all the curves follow the same trend. However, as heart rate increases, the curve size becomes larger. It can be observed that at the end of each curve, a stroke volume decreases. This reduction in stroke volume can indicate the blood backflow towards left ventricle [28]. As seen in Figure 4, the changes from heart rate of 60 to $80 \mathrm{bpm}$ are much lower than those between heart rate of 98 to $147 \mathrm{bpm}$ and the next ones [37-45].

Figure 5 provides the stroke work values during the ejection period while increasing the heart rate. A quadratic polynomial equation with a high accuracy $\left(\mathrm{R}^{2}=0.983\right)$ was fitted to the calculated data. The stroke work through exercise protocol increased by $121 \%$ from 60 to $125 \mathrm{bpm}$, and it did not increase much above $125 \mathrm{bpm}$. In a healthy subject, at higher heart rates the filling time of the left ventricle during diastole will decrease which brings down the preload and as a result of this the stroke volume will not show appropriate incrimination. This is because of the fact that the stroke volume does not increase considerably at the heart rate over $125 \mathrm{bpm}$ [27]. Stroke volume changes were followed by bigger increment after heart of 80 (Figure 4).

\section{Discussion}

\section{Study findings}

The study has investigated the initial use of a fluid-structure interaction model to calculate the stroke work done by the left ventricle by calculating the pressures and blood flow volume through the aortic valve. The hemodynamic data was acquired from a healthy subject while

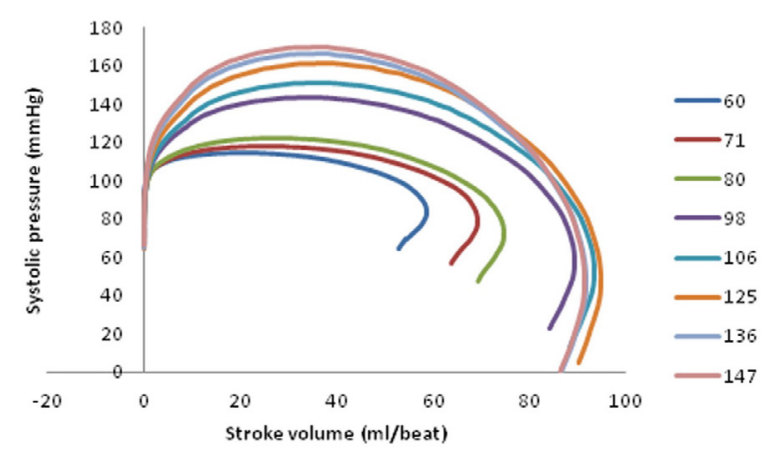

Figure 4: Systolic pressure changes to stroke volume at different heart rates.

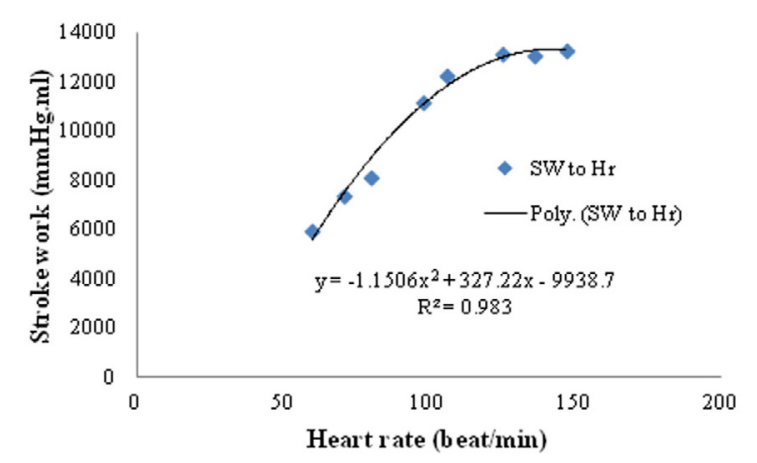

Figure 5: Stroke work changes to heart rate. exercising. Echo-Doppler-derived data provided essential information for simulation including aortic valve geometry and pressure boundary conditions. To the authors' knowledge, this would be the first time that exercise protocol measurements and FSI technique have been combined together to enable numerical estimations of stroke work. All the curves of systolic pressure changes to stroke volume at each heart follow the same pattern at different heart rates in terms of ascending and descending orders (Figure 4). To all appearances, stroke volume variations after heart rate 80 , is followed by an abrupt increase.

The FSI model predicted stroke work through exercise protocol to increase by $121 \%$ from 60 to $125 \mathrm{bpm}$, and then it did not increase much above $125 \mathrm{bpm}$. Our investigation provides initial insight as the feasibility of gaining a variety of conditions (e.g. changes due to exercise). The applicability of the modeling and its privileges such as low computational time and less expensive as compared to currently available clinical approaches, make it usable to estimate the stroke work in patients. As well as aforesaid benefits, our mathematical model has the capability to analyses different aortic geometries. Also, this model let patients to predict some situations which are not possible to clinically measure. To be more exact, based on the fact that the subject is human being applying such numerical model let physicians to understand and investigate probable conditions where patients could face in the future or on different circumstances [46-52].

Etiology of diseased states would be moreover assessable sine they may effect on shape and form (geometry) waveform and quantity of blood pressure (boundary conditions). On top of that, the vast majority of clinical techniques and research are not mechanical-based. Taking into consideration that the nature of heart valve functions is mostly mechanical-based, our discipline evidently steps toward to investigate the heart performance as it is a mechanical pump to a great extent.

\section{Clinical application and reliability}

Catheterization-Thermodilution is known as the golden method for measuring cardiac function [5] This is, however, an invasive technique. This has been proved such strategies are associated with potential perils including heart failure, death, and even cardiac arrhythmia [5]. The FSI modeling can offer clinical team information that is clinically compatible with Catheterization-Thermodilution and provide such data earlier and in a safer approach than traditional methods. Noninvasive measurement of the hemodynamic parameters and knowing the normal values for these parameters, the hemodynamic status of each patient can be represented as percent changes from the norm. This can result in numerical modeling of the hemodynamic status, and clinicians may be able to more accurately react to the individual needs of the patient with appropriate treatment. Furthermore, numerical simulations are capable to precisely estimate cardiac hemodynamics. This is mostly due to the fact that it does not deal with inter- and intra-observer validity variables which are mainly the argument for performing ECG. This kind of unevenness is contingent upon personal expertise and the image capture ability of the user. Therefore, the fundamental matter is to prove the credibility of modeling methods for cardiac evaluations. Moreover, clinical experiments performed on animal subjects. Amezcua et al. [12-18] demonstrated to avoid any risks for human subjects, can glorify the merits of numerical approaches.

\section{Comparison to the literature}

Figure 6 performs stroke work changes to mean arterial pressure (for the exercise protocol). As it can be observed, slope of the results reported by this paper $(168.08 \mathrm{mmHg} \cdot \mathrm{ml} / \mathrm{ml})$ and are within the reported clinical data varied from 46.56 to $335.05 \mathrm{ml}[1,2,4,5,12]$. The 


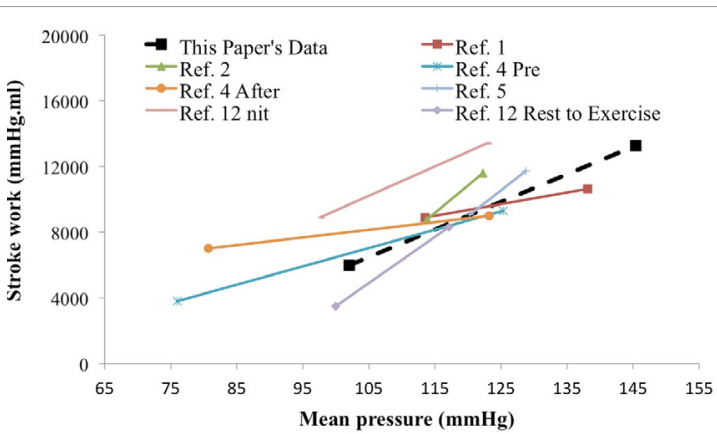

Figure 6: FSI and clinical stroke work changes to mean arterial pressure for the exercise protocol.

average slope of clinical data is $191.42 \mathrm{mmHg} \cdot \mathrm{ml} / \mathrm{ml}$. The reported results of this paper are within $12.2 \%$ with the literature reported data. The differences could be due to weight, sex, age and race of patients. It can be concluded that the reported data from the numerical model in this paper is in an acceptable range of reported clinical data.

\section{Limitations and future trends}

The limitations of this numerical study and future suggestions are listed as following:

1. Using two-dimensional rather than three-dimensional geometry.

2. Applying linear, homogenous and isotropic features for simplification of aortic valve's mechanical properties.

3. Mechanical properties are based on generalized information, and are not specific to the patient.

\section{Considering blood as a Newtonian and incompressible fluid.}

Despite model limitations, we fulfilled an acceptable consistency with the general literature.

Undoubtedly, a three-dimensional model may result in more precise predictions; however, it would also be raising the processing time (that is less than 15 minutes for the two-dimensional model). This should hold shortcomings for clinical applications.

\section{Conclusion}

We have presented a two-dimensional fluid-structure interaction simulation of aortic valve having the ability to predict stroke work from rest to exercise stage. Derived regression for stroke work changes versus mean arterial pressure showed the averagely $15 \%$ difference between numerical and clinical data. Thus, this model provides initial insights as the potential for predicting stroke work for a specific individual within a 15-minute response time that matches with clinical data.

\section{References}

1. Carrick-Ranson G, Doughty RN, Whalley GA, Walsh HJ, Gamble GD, et al. (2012) The larger exercise stroke volume in endurance-trained men does not result from increased left ventricular early or late inflow or tissue velocities. Acta Physiologica 205: 520-531.

2. Bouchard MJ, Denault A, Couture P (2004) Poor correlation between hemodynamic and echocardiographic indexes of left ventricular performance in the operating room and intensive care unit. Crit Care Med 32: 644-648.

3. Gunzinger R, Heimisch W, Augustin N (2005) Diastolic unloading and improved LV pump efficiency early after repair of the insufficient mitral valve. Thorac Cardiovasc Surg 53: 9-15.

4. Baba HA, Wohlschlaeger J, Stubbe HD (2004) Heat shock protein 72 and apoptosis indicate cardiac decompensation during early multiple organ failure in sheep. Intensive Care Med 30:1405-1413.

5. Lavdaniti M (2008) Invasive and non-invasive methods for cardiac output measurement. Int J Caring Sci 1:112-117.

6. Hofer CK, Ganter MT, Zollinger A (2007) What technique should I use to measure cardiac output? Curr Opin Crit Care 13: 308-317.

7. Engoren M, Barbee D (2005) Comparison of cardiac output determined by bioimpedance, thermodilution, and the Fick method. Am J Crit Care 14: 40-45.

8. Loeb HS, Rahimtoola SH, Rosen KM, Sinno MZ, Chuquimia R, et al. (1973) Assessment of ventricular function after acute myocardial infarction by plasma volume expansion. Circulation 47: 720-728.

9. Linhart JW (1971) Pacing-induced changes in stroke volume in the evaluation of myocardial function. Circulation 43: 253-261.

10. Hamosh P, Cohn JN (1971) Left ventricular function in acute myocardia infarction. J Clin Investigation 50: 523

11. Kamoi S, Squire D, Revie JA, Chase JG (2014) Accuracy of stroke volume estimation via reservoir pressure concept and three element windkessel model.

12. Amezcua JL, Palmer RMJ, Souza BD, Moncada S (1989) Nitric oxide synthesized from L-arginine regulates vascular tone in the coronary circulation of the rabbit. British J Pharmacol 97: 1119-1124.

13. Baydoun AR, Woodward B (1991) Effects of bradykinin in the rat isolated perfused heart: Role of kinin receptors and endothelium-derived relaxing factor British J Pharmacol 103: 1829-1833.

14. Brown IP, Thompson Cl, Belloni FL (1993) Role of nitric oxide in hypoxic coronary vasodilatation in isolated perfused guinea pig heart. American $J$ Physiol 264: H821-H821.

15. Lamontagne D, Pohl U, Busse R (1991) N G-nitro-l-arginine antagonizes endothelium-dependent dilator responses by inhibiting endothelium-derived relaxing factor release in the isolated rabbit heart. Pflügers Archiv 418: 266 270.

16. Park KH, Rubin LE, Gross SS, Levi R (1992) Nitric oxide is a mediator of hypoxic coronary vasodilatation. Relation to adenosine and cyclooxygenasederived metabolites. Circulation Res 71: 992-1001.

17. Smith RE, Palmer RM, Bucknall CA, Moncada S (1992) Role of nitric oxide synthesis in the regulation of coronary vascular tone in the isolated perfused rabbit heart. Cardiovascular Res 26: 508-512.

18. Ueeda M, Silvia, SK, Olsson RA (1992) Nitric oxide modulates coronary autoregulation in the guinea pig. Circulation Res 70: 1296-1303.

19. Al-Atabi M, Espino DM, Hukins DWL (2010) Computer and experimental modelling of blood flow through the mitral valve of the heart. J Biomech Sci Eng 5: 78-84

20. De Hart J, Peters GW, Schruers PJ, Baaijens FP (2000) A two-dimensiona fluid-structure interaction model of the aortic valve. J Biomech 33: 1079-1088.

21. De Hart J, Peters GW, Schreurs PJ, Baaijens FP (2003a) A three-dimensional computational analysis of fluid-structure interaction in the aortic valve. $J$ Biomech 36:103-112.

22. De Hart J, Baaijens FP, Peters GW, Schreurs PJ (2003b) A computationa fluid-structure interaction analysis of a fiber-reinforced stentless aortic valve. J Biomech 36: 699-712.

23. Espino DM, Shepherd DET, Hukins DWL (2012) A simple method for contact modelling in an arbitrary frame of reference within multiphysics software. $J$ Mech 29: N9 - N14

24. Espino DM, Shepherd DET, Hukins DWL (2013) Development of a transient large strain contact method for biological heart valve simulations. Comput Methods Biomech Biomed Engin 16: 413-424

25. Espino DM, Shepherd DET, Hukins DWL (2014) Evaluation of a transient simultaneous, Arbitrary Lagrange Euler based multi-physics method for simulating the mitral heart valve. Comput Methods Biomech Biomed Engin 17 : 450-458.

26. Stijnen JMA, De Hart J, Bovendeerd PHM, Van De Vosse FN (2004) Evaluation of a fictitious domain method for predicting dynamic response of mechanical heart valves. J Fluids Struct 19: 835-850

27. Bahraseman HG, Hassani K, Khosravi A, Navidbakhsh M, Espino DM, et al 
Citation: Bahraseman HG, Languri EM, Espino DM, Shojaei H, Hassani K, et al. (2017) Applied Fluid-Structure Interaction Technique to Initial Insight into the Effect of Exercise on the Aortic Valve Stroke Work. J Appl Mech Eng 6: 295. doi: 10.4172/2168-9873.1000295

Page 5 of 5

(2013) Estimation of maximum intraventricular pressure: A three-dimensional fluid-structure interaction model. Biomed Eng.

28. Bahraseman HG, Hassani K, Navidbakhsh M, Espino DM, Sani ZA, et al, (2014a) Effect of exercise on blood flow through the aortic valve: a combined clinical and numerical study. Comput Methods Biomech Biomed Engin 17: 1821-1834.

29. Bahraseman HG, Hassani K, Khosravi A, Navidbakhsh M, Espino DM (2014b) Combining numerical and clinical methods to assess aortic valve hemodynamics during exercise. Perfusion 29: 340-350.

30. Khosravi A, Bahraseman H, Hassani K, Kazemi-Saleh D (2014) Numerical method to measure velocity integration, stroke volume and cardiac output while rest: using 2D fluid-solid interaction model. Eng Solid Mech 2: 91-100.

31. Kuan MYS, Espino DM (2015) Systolic fluid-structure interaction model of the congenitally bicuspid aortic valve: Assessment of modelling requirements. Comput Methods Biomech Biomed Engin 18: 1305-1320.

32. Weinberg EJ, Kaazempur-Mofrad MR (2008) A multiscale computational comparison of the bicuspid and tricuspid aortic valves in relation to calcific aortic stenosis. J Biomech 41: 3482-3487.

33. Pedley TJ, Schroter RC, Seed WA, Parker KH (1978) The mechanics of the circulation. Oxford: Oxford University Press, UK.

34. Govindarajan V, Udaykumar HS, Herbertson LH, Deutsch S, Manning KB (2010) Two-dimensional fsi simulation of closing dynamics of a tilting disk mechanical heart valve. J Med Devices 4: 1-11.

35. Koch TM, Reddy BD, Zilla P, Franz T (2010) Aortic valve leaflet mechanical properties facilitate diastolic valve function. Comput Methods Biomech Biomed Engin 13: 225-234.

36. Formaggia L, Nobile F (1999c) East-West. J Numer Math 7: 105-132.

37. Murphy SL, Xu J (2012) Deaths: Preliminary Data for 2010 (1st edn). Atlanta: National Vital Statistics Reports.

38. Caro CG, Pedley TJ, Schroter RC, Seed WA (1978) The mechanics of the circulation. Oxford: Oxford University Press, UK.

39. Bellhouse BJ (1972) The fluid mechanics of heart valves. In: Cardiovascular fluid dynamics. Volume 1. Bergel DH (ed). London: Academic Press, UK.
40. Peskin CS (1972) Flow patterns around heart valves: a numerical method. J Comput Phys 10: 252-270.

41. Peskin CS (1977) Numerical analysis of blood flow in the heart. J Comput Phys 25: $220-252$.

42. Dowell EH, Hall KC (2001) Modelling of fluid-structure interaction. Annu Rev Fluid Mech 33: 445-490.

43. Wall W, Gerstenberger A, Gamnitzer P, Forster C, Ramm E (2006) Large deformation fluid-structure interaction-advances in ALE methods and new fixed grid approaches. In: Fluid-structure interaction. Bungartz HJ, Shafer M (eds.) Springer, Berlin, Germany.

44. Van De Vosse FN, De Hart J, Van Oijen CHGA, Bessems D, Gunther TWM et al. (2003) Finite-element-based computational methods for cardiovascular fluid-structure interaction. J Eng Math 47: 335-368.

45. Donea J, Giuliani S, Halleux JP (1982) An arbitrary Lagrangian-Eulerian finite element method for transient dynamic fluid-structure interactions. Comput Methods Appl Mech Engrg 33: 689-723.

46. Xia GH, Zhao Y, Yeo JH (2005) Numerical simulation of 3D fluid-structure interaction using AN immersed membrane method. Modern Physics Letters $B$ 19: $1447-1450$.

47. Peskin CS, Wolfe AW (1978) The aortic sinus vortex. In: Federation proceedings 37: $2784-2792$.

48. Schramm W (2010) The units of measurement of the ventricular stroke work: A review study. J Clin Monit Comput 24: 213-217.

49. Klabunde R (2011) Cardiovascular physiology concepts. Lippincott Williams \& Wilkins, USA.

50. Zubieta-Calleja G, Paulev PE (2004) Medical physiology and pathophysiology: Essentials and Clinical Problems. (2nd edn). Copenhagen Medical Publisher Denmark.

51. Lewis T (1915) Lectures on the heart. PB Hoeber. USA.

52. Grossman W (2006) Grossman's cardiac catheterization, angiography, and intervention. Lippincott Williams \& Wilkins, USA. 